\title{
ANÁLISIS DEL CONTEXTO SOCIO-ECONÓMICO, COMERCIAL, FINANCIERO E INTERNACIONAL DE LAS PYMES ECUATORIANAS.
}

\author{
ANALYSIS OF THE SOCIO-ECONOMIC, COMMERCIAL, FINANCIAL AND \\ INTERNATIONAL CONTEXT OF ECUADORIAN SMES.
}

Halder Yandry Loor Zambrano, Mgs. Magíster en Administración de Empresas, mención Dirección Financiera (Ecuador). Docente de la Universidad Técnica de Manabí, Ecuador. yandry.loor.zambrano@gmail.com

Daniela Marina Ureta Santana, Mgs. Magíster en Administración de Empresas (Ecuador). Docente de la Universidad Técnica de Manabí, Ecuador. damaursa@yahoo.com

Gonzalo Andrés Rodríguez Arrieta, Mgs. Magíster en Gestión Empresarial y en Dirección de Recursos Humano (Ecuador). Docente de la Universidad Eloy Alfaro de Manabí, Ecuador. profegonzalorodriguez@gmail.com

Evelyn Dyann Cano Lara, Ph.D. Doctora en Ciencias Administrativas (Perú). Docente de la Universidad Laica Eloy Alfaro de Manabí (ULEAM), Ecuador. evelyn.cano@uleam.edu.ec

\section{ARTÍCULO DE REFLEXIÓN}

Recibido: 12 de mayo de 2018.

Aceptado: 25 de junio de 2018.

\section{RESUMEN}

El propósito de este artículo es analizar la realidad de las pequeñas y medianas empresas (PYMEs) ecuatorianas, como motor principal del tejido productivo de la economía del país; 
durante el desarrollo del trabajo se efectuará un diagnóstico de la situación de esta tipología de empresas dentro del contexto social, económico, comercial, financiero e internacional en el que se desenvuelven.

La importancia del tema de investigación se ve reflejado en la participación del sector de la PYME en el desarrollo de la economía, así como al fomento del empleo directo e indirecto que genera. También se determinó la necesidad de la mejora que es necesaria en el sector a fin de poder internacionalizar sus productos y servicios, donde la incorporación de la innovación, la tecnología en todos sus procesos y el apoyo del crédito público y privado son de vital importancia.

En cuanto al método de investigación de base para este trabajo, es del tipo descriptivo, en tanto que a través de la revisión bibliográfica se busca describir la situación de las PYMEs del Ecuador y la caracterización actual de los cuatro parámetros objetos de estudio.

Palabras clave: PYMEs, Realidad, Contextualización, Socio - Económico, Comercial, Financiero e Internacional.

\section{ABSTRACT}

The purpose of this article is to analyze the reality of Ecuadorian small and medium enterprises (SMEs), as the main engine of the productive fabric of the country's economy; During the development of the work, a diagnosis will be made of the situation of this type of companies within the social, economic, commercial, financial and international context in which they operate.

The importance of the research topic is reflected in the participation of the SME sector in the development of the economy, as well as in the promotion of direct and indirect employment that it generates. It also identified the need for improvement that is necessary in the sector in order to internationalize their products and services, where the incorporation of innovation, technology in all its processes and the support of public and private credit are of vital importance. 
Regarding the basic research method for this work, it is of a descriptive type, while the literature review seeks to describe the situation of SMEs in Ecuador and the current characterization of the four parameters under study.

Keywords: SMEs, Reality, Contextualization, Socio - Economic, Commercial, Financial and International.

\section{INTRODUCCIÓN}

Reconocer la integridad de una PYME conlleva a analizar la complejidad de los elementos que deben ser considerados en su conceptualización y consecuente clasificación. Para Dini, Ferraro, y Gasaly (2007) al discutir sobre la definición única de lo que se considera PYME permitiría que "la capacidad de permanecer en el mercado dependa de sus recursos internos y de las potencialidades que aportan las modalidades de comunicación, relacionamiento e intercambio que las PYMEs establecen con otras empresas o entes del sistema productivo".

En algunos casos se observa que a nivel latinoamericano no existe uniformidad sobre los elementos comunes que lleven una definición estandarizada en los diferentes países, así como el entendimiento de los sectores y criterios utilizados en relación a las PYMEs. Contrariamente a lo que ocurre en la Unión Europea, donde existe y se utiliza una sola definición de PYME, los países latinoamericanos no cuentan con un acuerdo común sobre el concepto, los esfuerzos realizados para estandarizar los criterios, no han sido exitosos. Pocos lo han logrado y a nivel de países, la definición presenta variabilidad (Vives, Corral, \& Iñigo, 2005).

En un sentido general y tratando de simplificar en características lo que son las PYMEs Cardozo, Velásques de Naime y Rodriguez Monroy (2012, pp. 16-32) determinan que se trata de un tipo de organización mayoritariamente dirigida por el fundador o un familiar, que brinda un servicio o produce bienes, reconociendo un mercado de cobertura poco amplio en referencia a un país, y con poca cantidad de trabajadores por lo que su forma organizativa es simple y dependiente del propietario. 
Más allá de la diversidad de definiciones, sectores de participación y estructuras, las PYMEs tienen el protagonismo en la economía mundial porque la mayoría de empresas de países en vías de desarrollo, en países desarrollados y en países emergentes, pertenecen a esta tipología. Al ser la forma más predominante, son reconocidas como el motor del crecimiento económico futuro, son responsables de la mayor creación de puestos de trabajo y realizan importantes contribuciones a la innovación, productividad y crecimiento económico (Santos, 2013), a pesar de las desventajas de tamaño, estructura, productividad, financiamiento entre otros, que enfrentan con las grandes empresas. Su protagonismo se debe a la importancia que tienen en la economía de los países y por el alto grado de generación de empleo, según lo expuesto por Gómez, García, y Marín (2009, citado en García \& Villafuerte, 2015).

Según lo determina Agyapong (2010), las PYMEs integradas al aparato productivo como parte de la cadena de valor del aparato productivo de los países, coadyuvan en el impulso de todos los sectores de la economía. Dato que se corroboran en Latinoamérica al ver que casi el $95 \%$ de las empresas son PYMEs y que ellas generan casi el $65 \%$ de trabajo para la población latinoamericana (Savedra \& Hernadez, 2008).

En el ámbito de los países emergentes de Latinoamérica, Harvey y Wendel (2006) en un informe del Banco Mundial (BM), y Guaipatín (2003) en un reporte del Banco Interamericano de Desarrollo (BID), señalan el importante rol de las PYMEs en la región y manifiestan que el acceso al financiamiento bancario es un problema común y tiende a ser una característica de las naciones en desarrollo (García \& Villafuerte, 2015).

La República del Ecuador se ha visto inmersa en una serie de cambios que han venido transformando la forma en la que el mercado se comporta; en este contexto, las empresas ecuatorianas de menor tamaño, han debido ir evolucionando para adaptarse en forma rápida y eficiente a la nueva realidad de la economía ecuatoriana, pero no todas han logrado una transición exitosa (Prado, 2005). Con el afán de contribuir a la generación de mejores prácticas empresariales, se presenta a continuación, un análisis de la realidad de las PYMEs en el Ecuador, a partir de una visión objetiva de cuatro ámbitos de observación que refleja la situación de las mismas: socio-económico, comercial, financiero e internacional. La determinación de estos contextos denota la importancia de las pequeñas y medianas empresas en la economía ecuatoriana; asimismo, se tratarán 
ciertas particularidades que las caracterizan, pero sobre todo se abordarán la participación internacional que hacen de este tipo de empresas, entes claves para el fortalecimiento del aparato productivo del país.

Respecto a la metodología utilizada en este trabajo, es una investigación de tipo descriptiva, en base a una exhaustiva revisión bibliográfica, donde se expone literatura y estadística que muestran la relación existente entre los factores a estudiar y los resultados que evidencian el comportamiento actual de estas empresas; el diseño de la investigación servirá de guía para la validación de la información que certifiquen la autenticidad de este artículo, siendo el objetivo de éste, establecer dicha relación a partir de datos cualitativos (fuentes bibliográficas) y cuantitativos (porcentajes).

\section{REVISIÓN TEÓRICA}

\section{Realidad de las PYMEs ecuatorianas.}

Actualmente, la PYME está enfrentando momentos difíciles, y se desenvuelve entre ajustes presupuestarios por parte del gobierno central, debido al decrecimiento de la economía del país en el primer trimestre del año 2016 en un 3 \%, según el informe de diciembre 2016 del Banco Central del Ecuador (el Ecuador reportó tres trimestres consecutivos de decrecimiento, entre julio del 2015 y marzo del 2016). Se justifica la caída de la economía a causa de la caída del precio del petróleo, la apreciación del dólar estadounidense, la ejecución de las salvaguardias junto con las reformas tributarias, y el terremoto que azotó las provincias de Manabí y Esmeraldas el pasado 16 de abril de 2016, lo que ha incrementado los niveles de desempleo en un 5,7 \% a marzo de 2016 (en comparación con el 3,8 \% a marzo del 2015, un incremento de 1,9 puntos porcentuales) según la última Encuesta Nacional de Empleo, Desempleo y Subempleo (2016) presentada por el INEC, dificultando con ello aún más las posibilidades de internacionalización de muchas de ellas. En ese ámbito, las preferencias arancelarias que forman parte del Sistema General de Preferencias Plus, cesarán y esto contribuye a que este sector productivo (PYME) se encuentre en condiciones difíciles, pero no imposibles, para competir en el mercado europeo, norteamericano e incluso con países vecinos como Colombia y Perú (Basantes, 2016). Así también, las salvaguardias y sobre tasas arancelarias que modificaron las condiciones de intercambio comercial para los productos procedentes de Europa y otros 
países, se retrasaron al inicio del año 2016 y justo cuando se estableció un cronograma de desmontaje de las restricciones, el terremoto del 16 de abril de 2016 cambió el panorama drásticamente.

Según el Ministerio de Industrias y Competitividad del Ecuador, en lo que respecta a la organización jurídica de las PYMES, el 37,2 \% de ellas son Compañías Limitadas y destaca que un $35,2 \%$ de ellas operan como persona natural. Además, que el $97 \%$ de las PYMES posee Registro Único de Contribuyente (Ministerio de Industria y Competitividad, 2010).

Se pueden contar entre las actividades que realizan las PYMEs en el Ecuador las siguientes: "Comercio al por Mayor y al por Menor, Agricultura, Silvicultura y Pesca, Industrias Manufactureras, Construcción, Transporte, Almacenamiento, y Comunicaciones, Bienes Inmuebles y Servicios prestados a las empresas, Servicios Comunales, Sociales y Personales" (SRI, 2015).

Para el $44 \%$ de las empresas, el mercado es básicamente local, es decir la ciudad en la cual las empresas desarrollan sus productos, mientras que para el $26 \%$ el alcance es regional (ciudad, provincia de origen y provincias circunvecinas), el $8 \%$ tiene una cobertura hacia las provincias limítrofes con países vecinos y solamente el $6 \%$ realiza exportaciones (Ministerio de Producción, 2010).

Otro punto de análisis es el de la concentración y caracterización funcional de las PYMEs en el Ecuador, vista desde el punto al tipo de actividad que realiza, el $22 \%$ están vinculadas a la actividad comercial, recordando que en general, el comercio se lo puede entender como el cambio o transacción de algo a cambio de otra cosa de igual valor. Por actividades comerciales 0 industriales entendemos tanto intercambio de bienes o de servicios que se afectan a través de un mercader o comerciante, el comerciante es la persona física o jurídica que se dedica al comercio en forma habitual, como las sociedades mercantiles ${ }^{1}$ (SCIAN, 2002).

\footnotetext{
${ }^{1}$ El Sistema de Clasificación Industrial de América del Norte (SCIAN) fue desarrollado para generar estadísticas comparables entre los tres países de América del Norte, en un trabajo conjunto de sus dependencias gubernamentales. El objetivo es proporcionar un marco único, consistente y actualizado para la recopilación, análisis y presentación de estadísticas de tipo económico y que refleje la estructura económica de la economía mexicana (SCIAN, 2002).
} 
Tomando en cuenta estos datos, se toma información referencial del comportamiento de las PYMEs en el sector comercial. Considerando los datos emitidos por el INEC (2010), a partir de su último Censo Económico del año 2010, las empresas comerciales son en su gran mayoría, el motor que impulsa el aparato productivo del país teniendo a su vez el sector Comercio al por mayor y al por menor una representación del 54 \% a nivel nacional, evidenciando que un poco más de la mitad de las empresas se dedican al comercio en el Ecuador.

Este tipo de establecimientos centran su actividad primordialmente a la compraventa de productos industrializados, pero también de otros que provienen de la agricultura, pesca, extracción, entre otros, sin ejecución de ningún tipo de transformación, es decir el sector comercial sirve en muchas ocasiones de nexo con el consumidor final para las empresas del sector primario y secundario de la economía. EI INEC en su clasificador de actividades (generado a partir del Censo del año 2010), determina que el sector comercial tiene mayor cantidad de establecimientos en lo que se refiere a comercio al por menor, especialmente en lo que respecta a tiendas minoristas o también llamadas tiendas de barrio, las cuales ocupan el primer lugar en cuanto a cantidad de estas dentro del número total de establecimientos económicos del país, y que en su mayoría son unipersonales y de carácter familiar. Asimismo, es necesario destacar la relevancia del sector del comercio al por menor ya que se ve la necesidad de impulsar el desarrollo del comercio, para mejorar no solo las ventas y los ingresos económicos de las PYMEs, el sector comercio es un gran generador de empleo y es muy alta su contribución a los ingresos de gran cantidad de familias que son propietarios de estos negocios o en todo caso laboran como dependientes, pero sí hay que tomar en consideración que la totalidad de estos comercios constituyen PYMEs, inclusive muchas de estas son del orden micro empresarial e individual.

La elaboración de productos correspondientes a alimentos y bebidas constituye el 7,7 \% del Valor Agregado Bruto dentro del Producto Interno Bruto (PIB), representando el total de establecimientos que han declarado actividad económica; también, el 36,9 \% se dedica a actividades relacionadas con alimentos y bebidas, de estos, un 5,4 \% está relacionado con la elaboración de productos alimenticios (INEC, 2010).

Tomando en consideración este antecedente, en la Tabla 1 se presenta el número de establecimientos a nivel nacional que forman parte del sector del comercio al por menor 
clasificadas según su actividad económica (razón por la que sólo se han considerado 10 de ellas):

Tabla 1. Actividades económicas principales.

\begin{tabular}{|l|c|c|}
\hline \multirow{2}{*}{$\begin{array}{l}\text { Venta al por menor en comercios no especializados con } \\
\text { predominio de la venta de alimentos, bebidas y tabaco }\end{array}$} & 87244 & $17,4 \%$ \\
\cline { 2 - 3 } & 44722 & $8,9 \%$ \\
\hline Actividades de restaurantes y de servicio móvil de comidas. & 25432 & $5,1 \%$ \\
\hline Venta al por menor de prendas de vestir, calzado y artículos & 20437 & $4,1 \%$ \\
\hline Mantenimiento y reparación de vehículos automotores. & 17528 & $3,5 \%$ \\
\hline Otras actividades de telecomunicaciones & 15627 & $3,1 \%$ \\
\hline $\begin{array}{l}\text { Venta al por menor de alimentos, bebidas y tabaco en } \\
\text { puestos }\end{array}$ & 15474 & $3,1 \%$ \\
\hline $\begin{array}{l}\text { Otras actividades de venta al por menor en comercios no } \\
\text { especializado }\end{array}$ & 14634 & $2,9 \%$ \\
\hline $\begin{array}{l}\text { Venta al por menor de alimentos en comercios } \\
\text { especializados. }\end{array}$ & 14426 & $2,9 \%$ \\
\hline Actividades de peluquería y otros tratamientos de belleza. & 10884 & $2,2 \%$ \\
\hline $\begin{array}{l}\text { Venta al por menor de productos farmacéuticos y } \\
\text { medicinales }\end{array}$ & 233809 & $46,7 \%$ \\
\hline Los 229 sectores restantes & & \\
\hline
\end{tabular}

Fuente: Censo Nacional Económico 2010. Elaborado por el Observatorio de la PYME de Universidad Andina Simón Bolívar.

Es decir, su contribución al Producto Interno Bruto (PIB) y al empleo, principalmente por la cantidad de empresas que están incluidas dentro de este sector comercial, más no por el tamaño de éstas. Es importante destacar la participación que ha tenido dentro del sector comercio al por menor, específicamente la división G47² (Comercio al por menor, excepto el de vehículos automotores y motocicletas) según la revisión del CIIU33 (Clasificación Industrial Internacional Uniforme) revisión $4^{4}$ en la economía ecuatoriana, su evolución

\footnotetext{
${ }^{2}$ División 47: Empresas correspondientes al sector del comercio al por menor, excepto el de automotores y motocicletas según el CIIU, revisión 4.

${ }^{3}$ CIIU: Clasificación Internacional industrial uniforme de todas las actividades económicas.

${ }^{4}$ Revisión 4: Revisión examinada y aprobada por la Comisión de Estadística en Marzo 2006, como la norma internacionalmente aceptada. Sustituye ahora a la tercera revisión de la clasificación 3 de la CIIU.
} 
durante los últimos años, así como la participación que han tenido las PYMEs en dicho sector (Directorio de Empresas y Establecimientos, 2014).

El sector del comercio en el Ecuador y según la nueva clasificación internacional industrial uniforme (CIIU 4) está codificado con la categoría G (Comercio al por menor excepto el de vehículos automotores y motocicletas); este sector siguiendo lo determinado por el Directorio de Empresas y Establecimientos (2014) está compuesto por las siguientes categorías:

> G45: Comercio al por mayor y al por menor, reparación de vehículos automotores y motocicletas.

$>$ G46: Comercio al por mayor, excepto el de vehículos automotores y motocicletas.

$>$ G47: Comercio al por menor, excepto el de vehículos automotores y motocicletas

El sector G47 se divide en otros subsectores siguiendo a la consideración de la Superintendencia de Compañías (2010):

- G471: Venta al por menor en comercios no especializados.

$>\quad$ G472: Venta al por menor de alimentos, bebidas y tabaco en comercios especializados.

$>\quad$ G473: Venta al por menor de combustibles para vehículos automotores en comercios especializados.

> G474: Venta al por menor de equipo de información y de comunicaciones en comercios especializados.

$>\quad$ G475: Venta al por menor de otros enseres domésticos en comercios especializados

$>\quad$ G476: Venta al por menor de productos culturales y recreativos en comercios especializados.

> G477: Venta al por menor de otros productos en comercios especializados.

$>\quad$ G478: Venta al por menor de puestos de venta y mercados.

$>\quad$ G479: Venta al por menor no realizada en comercios, puestos de venta o mercados. 
El sector G47 en el Ecuador está integrado por 232.760 establecimientos económicos, de un total de 500.217 que declararon actividad económica en el Censo Nacional Económico del año 2010, es decir, el 46,53 \% del total. En este sector se contemplan las actividades económicas relacionadas a la venta de: alimentos, bebidas y tabaco (50,5\%); otras actividades de comercio al por menor (23,3\%); prendas de vestir, calzado y artículos de cuero (10,9\%); productos farmacéuticos y medicinales, cosméticos y artículos de tocador $(4,7 \%)$; libros, periódicos y artículos de papelería (3,2\%); y, aparatos eléctricos de uso doméstico, muebles y equipo de iluminación (3,0 \%) (INEC, 2010).

El diagnóstico general presentado hasta aquí, respecto de lo que acontece con las PYMEs del Ecuador, conlleva a presentar a continuación un análisis del contexto socio-económico, comercial, financiero e internacional de este tipo de empresas, de acuerdo al objetivo del presente trabajo.

\section{Contexto socio-económico.}

La PYME en el nuevo contexto mundial se enfrentan a nuevos desafíos bajo un mundo más globalizado y competitivo, obligándose a la incorporación de tecnología y la innovación como mecanismos competitivos y de creación de valor para el consumidor de sus productos o servicios (Alburquerque, 1997). Lo anterior cambia las estructuras de los procesos y las personas necesarias para esta adaptación haciendo por ello necesario un cambio adaptativo social de la fuerza productiva del país (Arce \& Calves, 2018).

Cada uno de los cambio que la sociedad recibe por parte del crecimiento del sector empresarial determina una mejoría en la sociedad, en forma directa como es el empleo directo e indirecto que se crea (a través de la dependencia de productos o materias primas entre los sectores que aportan a un sector), así como indirecta como es el aumento de las rentas del estado vía cobro de impuesto y el gasto que habilita este aumento de las arcas del país (Cleri, 2013).

Betancourt y Jácome (2011) determinan que "el montaje de la estructura productiva que mantengan estas actividades industriales, requerirá la formalización de la PYME", que 
determina la formalización como tipo organizacional, la inclusión dentro de los mecanismos de apoyo al sector y la mejoría del trabajo informal.

El soporte a la sociedad y la economía de las PYMEs crea un soporte hacia el objetivo fundamental del Ecuador que es el buen vivir. Una definición importante es la que proporciona la Secretaría Nacional de Planificación y Desarrollo (SENPLADES), organismo que considera al trabajo, entre otras cosas, como aquello que no solamente permite el acceso de un ingreso para garantizar los bienes que permiten la reproducción de la vida con dignidad e independencia, sino que coadyuva al proceso de construcción de ciudadanos (Sánchez, 2012).

\section{Contexto comercial.}

Se conoce que las micro, pequeñas y medianas empresas (MIPYMEs), son la base del sistema comercial y de servicio de los países; de hecho, Ecuador está lleno de pequeñas empresas y se nutre diariamente de personas emprendedoras que deciden dejar la estabilidad o inestabilidad de sus trabajos para emprender un negocio propio; sin embargo, existe todavía muy poca difusión y conocimiento sobre la importancia de las PYMEs en la economía ecuatoriana.

Las PYMEs representan el 9,9\% de las unidades productivas y tienen una participación del $26,7 \%$ de las ventas totales sobre todos los tipos de empresas. Adicionalmente mantienen una participación sobre el total de los empleados afiliados para todos los tamaños de empresas del 39,1\% (INEC, 2014).

De acuerdo al último Censo Nacional Económico del INEC (2010), se indica que el 2,2 \% y $5,1 \%$ de pequeñas y medianas empresas, respectivamente, buscan mercados de otros países a la hora de comercializar los bienes y/o servicios que son generados en territorio ecuatoriano, lo cual se convierte en un indicador de que en el medio de la internacionalización de las PYMEs del Ecuador y a pesar de que algunas ya han experimentado el relacionamiento con compradores internacionales, todavía hay un camino por recorrer, en donde la articulación basada en el diálogo y acción público-privada se convierta en el soporte sobre el cual se levante una estrategia nacional dirigida al 
potenciamiento de la capacidad exportadora de la empresa ecuatoriana (Araque \& Argüello, 2015).

\section{Contexto financiero.}

El sector financiero en el Ecuador asienta sus bases tanto en el sector de la banca pública como en la privada; se ha evidenciado que la banca pública orienta sus recursos en mayor medida al mediano y largo plazo, mientras que la banca privada se concentra en el corto plazo, lo que implica que la banca pública financia más proyectos de inversión y productivos (el crédito productivo PYMEs en la banca pública representa el 6,35 \% y en el sistema financiero privado asciende al 0,5\%), mientras que la banca privada está dirigida mayoritariamente al financiamiento de capital de trabajo (financiamiento de inventarios y cuentas por cobrar). La colocación por distribución geográfica demuestra que la banca privada tiende a concentrar sus colocaciones en las principales ciudades (Quito y Guayaquil), mientras que la banca pública registra una participación más equilibrada (Neira, 2016).

Lasio, Arteaga y Caicedo (2010) relacionaron a las PYMEs con el sector financiero, determinando uno de los pilares fundamentales tanto para la persona que comienza su negocio como para el avance de la empresa. Los conceptos de micro empresario, emprendedor y emprendimiento van de la mano con el de la PYME, considerándose esta última como fruto de un emprendedor. Este tipo de empresario ávido de crecimiento y desarrollo requieren para llevar adelante sus propuestas de valor de un sector financiero que esté dispuesto al apoyo a nuevos negocios, sin historial crediticio muchas veces. Por lo que el crédito juega un rol fundamental, pero la racionalización de este en ocasiones suele ser excluyente, sobre todo con sectores amplios de prestatarios, entre los que se encuentran las micro, pequeñas y medianas empresas, ya que es un sector considerado como de alto riesgo. Las restricciones a un sector emprendedor terminan afectando a la economía del país, de acuerdo a Betancourt \& Jácome (2011) "coartar el fomento de actividades de comercio, de producción y de servicios; situación que atenta contra el desarrollo socioeconómico de un país".

Como destaca Neira (2016), no existe un plan o estrategia integral específica de inclusión financiera de las PYMEs con objetivos bien formulados, metas claras, marcos temporales 
y planes de acción. Sin embargo, el Plan Nacional del Buen Vivir 2013-2017, la Estrategia Nacional para el Cambio de la Matriz Productiva, la Agenda Sectorial de la Política Económica y la Agenda Sectorial para la Transformación Productiva cuentan con varios lineamientos de política orientados a la inclusión financiera de las PYMEs, por ejemplo, se hace mención a un sistema nacional de garantías, fondo de garantías, garantías recíprocas, mecanismos alternativos de fondeo para enfrentar el descalce de plazos, instrumentos de capital de riesgo, fondo de capital semilla, línea de crédito de largo plazo para compra de activos fijos a cargo de la Corporación Financiera Nacional (CFN), reducción de las tasas de interés para los segmentos de microcrédito y PYMEs, constitución de un mercado alternativo para la negociación de acciones de PYMEs y organizaciones de la economía popular y solidaria (Registro Especial Bursátil) y factoraje para obtener liquidez de corto plazo y capital de trabajo.

La insuficiente asignación de los recursos por parte de la banca privada a través de los procesos de intermediación financiera y la consabida incorporación de prácticas de racionamiento del crédito a sectores desatendidos de la sociedad, motivó a que el Estado ecuatoriano, a través de la banca pública, se insertara activamente dentro del sector financiero, tratando de ser un apoyo al crecimiento de la sociedad a través de bancos como Banco del Pacifico, o BanEcuador, o en sectores de mayor necesidad financiera a través de la Corporación Financiera Nacional.

\section{Contexto internacional de las PYMEs.}

La internacionalización vista como un proceso según Delgadillo de Lira (2004), implica el desarrollo de formas de comunicación, en las que la tecnología y el mundo virtual se entrelazan para facilitar procedimientos y abaratamiento de costos, donde el transporte juega un papel fundamental, ya que debe ser ágil y oportuno, presuponiendo en la mayoría de casos la eliminación de fronteras, lo que achica distancias entre países y convierte al mundo cada vez más global, pequeño y accesible, todo ello dentro de un marco de alta competencia.

La intervención de la PYME en el comercio exterior también es importante, es un beneficio para la economía del país porque las exportaciones crean trabajo, estimulan el crecimiento económico y mejoran la balanza de pagos; también ofrecen beneficios a largo plazo para 
la PYME, tales como el aumento de ingresos por ventas, la reducción del riesgo a partir de la diversificación de mercados, las economías de escala a partir del aumento de la producción, el aumento de competitividad y una mejora del conocimiento del mercado global y de la competencia (Hilton, 2005).

Pese a que la evidencia de su internacionalización es cada vez más palpable, las PYMEs no suelen tener mucha representación en el comercio mundial. En total, se estima que contribuyen entre un $25 \%$ y un $35 \%$ del total de las exportaciones de manufacturas. Aproximadamente, un quinto de las PYMEs del sector de manufacturas en los países miembros de la Organización para la Cooperación y el Desarrollo Económico (OCDE) tienen entre un $10 \%$ y un $40 \%$ de su volumen de facturación de operaciones internacionales (OCDE, 2005). Las PYMEs, tal como se conciben actualmente, se asocian a características distintivas de crecimiento, innovación e internacionalización, como formas de garantizar su permanencia en el tiempo y de generar estabilidad a sus socios y empleados.

Entre los productos exportados por las PYMEs ecuatorianas, se destaca el segmento de alimentos y bebidas, puesto que alrededor del $60 \%$ de sus exportaciones hacia la Unión Europea (UE) corresponden al segmento de frutas, legumbres y plantas; un $19 \%$ a productos de cacao, café y té; $15 \%$ a pescado y sus productos, mientras que el restante 6 $\%$ abarca otros productos, la mayoría de ellos del ámbito no alimenticio, entre estos se destacan artículos textiles, productos de madera y papel, bebidas espirituosas y tabaco, productos agropecuarios, productos de cuero, entre otros; en caso de no haberse dado el acuerdo con la UE, las afectaciones que podrían haber caído principalmente en el empleo, tomando en cuenta que este tipo de empresas son intensivas en la generación de mano de obra en Ecuador (Basantes, 2016).

Según lo expuesto, se ha tratado dar una visión general sobre este importante tema de internacionalización y se ha podido establecer que, así como hay empresas que pueden internacionalizarse, son muchos los motivos que pueden actuar como impedimentos para la actividad exportadora de las PYMEs del Ecuador. Entre las trabas para llegar al mercado internacional se encuentran percepciones y actitudes frente al riesgo de sus propietarios, al ser ellos los decisores son claves para que sus negocios puedan internacionalizarse; además, la falta de información y conocimiento acerca de las oportunidades que se 
presentan en los mercados extranjeros es una de las limitantes que no permiten que empresas con gran solidez puedan comercializar sus productos en otros mercados (Aerts, 1994).

También, aún en el caso de haber sido capaz de detectar las oportunidades en el exterior, la empresa puede encontrarse con problemas derivados con el desconocimiento de las costumbres, lenguaje, la legislación o las prácticas comerciales locales. Otra limitante importante para la internacionalización y que en este caso afecta en mayor medida a las PYMEs, es la falta de recursos y capacidades, así como los trámites burocráticos necesarios para iniciar el proceso de internacionalización; y, ciertos "papeleos" ocupan recursos valiosos para la empresa, uno de ellos es el tiempo.

De igual manera, en determinados países pueden existir normativas o regulaciones legales específicas a ciertos sectores que deberán cumplir obligatoriamente las empresas que deseen entrar a competir con ellos, como por ejemplo los estándares técnicos, las normativas de seguridad, las normativas de prueba o certificación de productos y procesos o las exigencias en cuanto a la puesta en el mercado del producto, a esto se refiere al etiquetado y distribución. Otra limitante para la exportación es la falta de contactos en los mercados exteriores y en especial el diferente acceso a canales de distribución adecuados en esos mercados, el disponer de distribuidores eficaces y sobre todo en los que la empresa pueda confiar es un aspecto clave en la expansión internacional, ya que sin ellos los productos ofertados no llegarán de forma adecuada a los consumidores. Todo ello limita las posibilidades de expansión internacional exitosa; finalmente, la falta de información puede extenderse hasta el punto de no hallarse al corriente de los programas gubernamentales y privados, respecto de la promoción y/o apoyo e impedir el proceso de internacionalización de PYMEs ecuatorianas con potencial exportador.

\section{ANÁLISIS DE LOS RESULTADOS}

\section{Potencial de exportación de las PYMEs ecuatorianas.}

Algo que merece atención especial, es la catástrofe que afectó a una parte de la población del Ecuador, entre ellas la provincia de Manabí y Esmeraldas, que no solo debilita al sector 
de las PYMEs en sus niveles de ventas, sino que a ello se le puede sumar su reubicación geográfica actual, razón por la que se dificulta aún más el hecho de que empresas sólidas y con todo el potencial productivo no formen parte del proceso exportador del país. Con la firma del Tratado Multipartes que el Ecuador firmó con la Unión Europea se pudo abrir las puertas para exportar a Europa y generar empleo, ya que los países que forman parte de este bloque comercial son el principal destino para las exportaciones no petroleras (aún a sabiendas que Reino Unido a esta fecha decidió, vía referéndum, salir del mencionado bloque), en este caso las PYMEs son las más beneficiadas, conociéndose de antemano que más de 1400 empresas de todo tamaño participan en las exportaciones hacia la UE, de las cuales más del $60 \%$ corresponden al segmento de MIPYMEs, según datos de la Federación Ecuatoriana de Exportadores (FEDEXPOR), en el caso de las microempresas, ese bloque representa un $47 \%$; para las PYMEs el $46 \%$ y las personas naturales un 44 $\%$.

Para el fortalecimiento de este tipo de emprendimientos en el mercado europeo, es de gran importancia el logro del tan anhelado acuerdo comercial, por lo que si las empresas quieren ganar consumidores en estas condiciones, es un nuevo reto al que deberán apostar, ya que mejorar su productividad sería unas de las acciones inmediatas a seguir e involucra la optimización de costos para poder ofrecer productos y servicios novedosos que no solo ayuden a regenerar el sector de las PYMEs sino a la economía del Ecuador (Basantes, 2016).

La conferencia de Naciones Unidas sobre Comercio y Desarrollo sostiene que las exportaciones de las PYMEs en los países en vías de desarrollo, emergen como una combinación, tanto del comportamiento de elementos del entorno interno (capacidades de organización interna) y externo en que se desenvuelven, así como de las características cualitativas y cuantitativas de los productos que buscan comercializar en mercados internacionales (Araque, 2015).

Araque (2015) analizó el potencial exportador para el perfil actual de la economía ecuatoriana dolarizada, determinándolo fundamental para el aumento de la cantidad de dólares en el país. A su vez la misma constitución (art. 304) determina la importancia del impulso al comercio fuera del Ecuador para el crecimiento de las economías regionales, siendo las empresas pequeñas y medianas las que más aportarían. 
Existen PYMEs que se destacan también por los productos gourmet dentro del reconocido mercado del lujo a nivel internacional, tratando en lo posible de demostrar potencialidades que tienen los productos gourmet ecuatorianos, en donde el éxito es combinar productos estrellas como el chocolate, el atún o el camarón para que importadores, distribuidores y representantes de supermercados vean las amplias posibilidades de los alimentos del país andino.

\section{CONCLUSIONES}

Sin lugar a duda queda entendida la importancia del papel que desempeñan las PYMEs en la economía ecuatoriana ya que no solo tienen una participación e incidencia en los diferentes ámbitos del país, sin embargo, es necesario que se tenga conciencia de lo que pasa en la actualidad con estas empresas, básicamente tomando en consideración las fuentes citadas. Para que las PYMEs puedan fortalecer el trabajo que realizan, es necesario mejorar el nivel tecnológico, esto en cuanto al proceso de producción, maquinaria, equipos y sobre todo mejorar sus endebles estructuras organizacionales, y esto se evidencia en el análisis del contexto financiero de ellas, donde es fundamental la disponibilidad de líneas de crédito que permitan realizar una inversión tecnológica, lo que lógicamente ayudará sustancialmente a la calidad de los productos o los servicios, teniendo como meta y referencia los estándares internacionales que manejan ciertas PYMEs a escala mundial.

Mucho se habla de la especialización, pero poco se hace al respecto; para las PYMEs, que son como se expresaba en el estudio, el motor del tejido productivo del país, es punto clave la formación y preparación del emprendedor ecuatoriano, debiéndose impulsar estos negocios porque existe un potencial en ellos y deben estar respaldados por un verdadero plan estratégico de comercialización. Ya es hora de abolir aquel pensamiento que es el cliente quien tiene que adquirir un producto o servicio por su cuenta, sino que son los emprendedores quienes deben ser lo suficientemente competentes como para incursionar en un mercado ampliamente globalizado en donde las necesidades del cliente crecen y donde los recursos son escasos. Con esto, se hace énfasis en que las PYMEs deben estar a la vanguardia y ser capaces de superar limitaciones internas, entonces, solo así una empresa podría estar preparada y ser competitiva en el mercado externo. 
Estos desafíos son precisamente los que deben enfrentar las PYMEs ecuatorianas; sin embargo, es compromiso de todos: universidades, gremios empresariales, los empresarios de forma individual, el gobierno central, organizaciones no gubernamentales, entre otros involucrados en ésta temática crucial para el desarrollo de la economía del país, promover el mejoramiento continuo de las capacidades distintivas de cada una de estas unidades empresariales, de tal forma que su desempeño productivo pueda ser altamente competitivo y atractivo no sólo dentro del mercado local y nacional, sino también para los mercados internacionales.

\section{REFERENCIAS BIBLIOGRÁFICAS}

Aerts, R. (1994). Becoming International: Benefits and pitfalls for entrepreneurial SMEs: Experiences from Belgium. England: Venciana.

Agyapong, D. (2010). Micro, small and medium enterprises activities, income level and poverty reduction in ghana. A synthesis of related literature. International Journal of Business and Management.

Alburquerque LLorens, F. (1997). Fomento productivo municipal y gestión del desarrollo económico local. Naciones Unidas, CEPAL. Santiago de Chile: CEPAL. Retrieved from https://repositorio.cepal.org/handle/11362/30780

Araque, W. (2015). Potencial de exportación de las Pymes. Revista Gestión(251), 28-32.

Araque, W., \& Argüello, A. (2015). Caracterización del proceso de internacionalización de las PyME ecuatorianas. FIR - FAEDPYME International Review, IV(7), 34-46.

Arce Castro, B., \& Calves Hernández, S. (2018). LA EVALUACIÓN DE LAS CADENAS DE VALOR COMO ESTRATEGIA PARA LA COMPETITIVIDAD DE LAS PYMES. Tecsistecat/(5). Retrieved from http://www.eumed.net/rev/tecsistecatl/n5/acch.pdf

Banco Central del Ecuador. (2016). Boletín de Información Estadístico Mensual: Diciembre 2016. Quito: Banco Central del Ecuador.

Basantes, X. (2016). Los tropiezos con la UE perjudican a las pymes. Diario El Comercio.

Betancourt, M., \& Jácome, H. (2011). Desigualdad en el acceso al crédito. In A. Serrano, ¡A (Re)distribuir! Ecuador para Todos. Quito.

Cardozo, E., Velásques de Naime, Y., \& Rodriguez Monroy, C. (2012). El concepto y la clasificación de PYME en América Latína. Global Conference on Business and Finance Proceedings, 7(2), 1630-1641. Retrieved from http://oa.upm.es/10940/2/Actas_del_Congreso_theibfr_CardozoNaimeMonroy\%5B $1 \% 5 \mathrm{D} . \mathrm{pdf}$ 
Cleri, C. (2013). El libro de las pymes. Buenos Aires: Granica.

Delgadillo de Lira, L. (2004). La internacionalización de las empresas familiares españolas en México: Análisis de sus factores determinantes. In J. M. Álvarez, Unión Europea y América Latina. Claves para un acercamiento (pp. 91-108). Madrid (España): Vision Net.

Dini, M., Ferraro, C., \& Gasaly, C. (2007, Noviembre). PYMES y articulación productiva. Resultados y lecciones a partir de experiencias en América Latina. Santiago de Chile, Chile.

García, G., \& Villafuerte, M. (2015). Las restricciones al financiamiento de las PYMES del Ecuador y su incidencia en la política de inversiones. Actualidad Contable Faces, 18(30), 49-73.

Gómez, A., García, D., \& Marín, S. (2009). Restricciones a la financiación de la pyme en México: una aproximación empírica. México: Universidad Autónoma Metropolitana - Azcapotzalco.

Guaipatín, C. (2003). Observatorio MIPYME compilación estadística para 12 países de la región. Informe de trabajo. División de micro, pequeñas y medianas empresas.

Washintong DC: Banco Interamerciano de Desarrollo.

Harvey, M., \& Wendel, C. (2006). SME Scoring: Key Inititatives,Opportunities and Issues. Retrieved from Boletín Wold Bank No - 38995: http://documents. worldbank.org/curated/en/2006/03/7448039/sme-credit-scoring-keyinitiativesopportunities-issues

Hilton, G. (2005). Knocking down export barriers to smaller firms. Business and Economic Review, 51(4), 18-20.

INEC. (2010). Instituto Nacional de Estadísticas y Censos. Retrieved from http://www.inec.gob.ec/archivos_temporales_descarga/AnuarioDESAE_2013.pdf

INEC. (2014). Instituto Nacional de Estadísticas y Censos.

INEC. (2016). Encuesta Nacional de Empleo, Desempleo y Subempleo. Indicadores Labores - Marzo 2016. Retrieved from http://www.ecuadorencifras.gob.ec/documentos/web-inec/EMPLEO/2016/Marzo2016/Presentacion\%20Empleo_0316.pdf

Lasio, V., Arteaga, E., \& Caicedo, G. (2010). Global Entrepreneurship Monitor Ecuador 2010. Retrieved from ESPAE - Graduate School of Management: http://www.espae.espol.edu.ec/wpcontent/uploads/2011/12/Reporte_GEM_2010.pdf

Ministerio de Industria y Competitividad. (2010). Caracteristicas básicas de las Pymes. Retrieved from www.industrias.gob.ec 
Ministerio de Producción. (2010). Mercados de las Pymes. Retrieved from www.produccion.gob.ec

Neira, S. (2016). Inclusión financiera de las pymes en el Ecuador. In N. U. CEPAL, FINANCIAMIENTO PARA EL DESARROLLO (pp. 1-49). Santiago: Naciones Unidas.

Prado, J. J. (2005). Pymes y Microempresas: Entre obstáculos y oportunidades.

Sánchez, J. (2012). Equidad y Politicas sociales. Algunas reflexiones para el caso ecuatoriano. In A. Serrano, ¡A (Re)distribuir! Ecuador para Todos (pp. 335-383). Quito.

Santos, L. (2013). Factores de éxito en la internacionalizacion de la empresa de los muebles de Andalucía. Retrieved from http://helvia.uco.es/xmlui/handle/10396/11794

Savedra, M., \& Hernadez, Y. (2008). Caracterización e importancia de las MIPYMES en Latinoamérica: Un estudio comparativo. Actualidad Contable Faces, 11(17), 122134. Retrieved from http://www.redalyc.org/articulo.oa?id=25711784011

SRI. (2015). ¿Qué son las PYMES? Retrieved from Servicio de Rentas Internas: http://www.sri.gob.ec/de/32

Vives, A., Corral, A., \& Iñigo, I. (2005). Responsabilidad social de la empresa en la PYMES de Latinoamérica. 1300 New York Avenue N.W.: Banco Interamericano de Desarrollo. 